\title{
Effects of the orientation of moving objects on the perception of streaming/bouncing motion displays
}

\author{
TAKAHIRO KAWABE and KAYO MIURA \\ Kyushu University, Fukuoka, Japan
}

\begin{abstract}
In this study, we examined the contribution of the orientation of moving objects to perception of a streaming/bouncing motion display. In three experiments, participants reported which of the two types of motion, streaming or bouncing, they perceived. The following independent variables were used: orientation differences between Gabor micropatterns (Gabors) and their path of motion (all the experiments) and the presence/absence of a transient tone (Experiment 1), transient visual flash (Experiment 2), or concurrent secondary task (Experiment 3) at the coincidence of Gabors. The results showed that the events at coincidence generally biased responses toward the perception of bouncing. On the other hand, alignment of Gabors with their motion axes significantly reduced the frequency of bounce perception. The results also indicated that an object whose orientation was parallel to its motion path strengthened the spatiotemporal integration of local motion signals along a straight motion path, resulting in the perception of streaming. We suggest that the effect of collinearity between Gabors and their motion path is relatively free from the effect of attention distraction.
\end{abstract}

A fundamental task of the perceptual system is to spatially and temporally identify external objects. In other words, it is necessary for the perceptual system to discriminate which objects in a present scene correspond to objects in the next scene. So far, research has suggested that dynamic changes in visual objects are detected by several visual mechanisms, such as low-level motion sensors (Adelson \& Bergen, 1985; Lu \& Sperling, 2001), attentive tracking (Cavanagh, 1992; Pylyshyn \& Storm, 1988; Verstraten, Cavanagh, \& Labianca, 2000), and spatiotemporal integration of moving objects (Alais \& Lorenceau, 2002; Gepshtein \& Kubovy, 2000).

The visual ability to identify objects in space and time has been assessed by examining perception, using ambiguous, bistable motion displays, a good example of which is a streaming/bouncing motion display in which two identical objects move toward each other, coincide, and then move away from each other. In this display, either of two types of motion is perceived; streaming, where each object continues to move along its own trajectory, or bouncing, where the two objects change trajectories as though colliding with each other at the point of coincidence (Bertenthal, Banton, \& Bradbury, 1993; Goldberg \& Pomerantz, 1982; Sumi, 1995). In general, streaming

This research was partly supported by the Japan Society for the Promotion of Science. We greatly appreciate Freya Cooper (Edanz editing) and Md. Kamal Uddin for correcting our English expressions. We are also deeply grateful to Nicholas R. Burns and two anonymous reviewers for critical and helpful comments on an earlier version of the manuscript. Correspondence concerning this article should be addressed to T. Kawabe, Department of Psychology, Faculty of Letters, Kyushu University, 6-19-1, Hakozaki, Higashi-ku, Fukuoka 812-8581, Japan (e-mail:kawabe@usi.kyushu-u.ac.jp). motion is dominantly perceived, perhaps because local motion integration through temporal motion recruitment occurs more easily along the same trajectory than between different trajectories (Anstis \& Ramachandran, 1986; Watamaniuk, McKee, \& Grzywacz, 1995).

Previous studies have reported that auditory signals can alter visual perception of streaming/bouncing displays. Sekuler, Sekuler, and Lau (1997) found that the perception of bouncing became dominant when a transient sound was presented at the coincidence of moving objects (hereafter, we will simply refer to this as at coincidence). They suggested that auditory signals at coincidence might be utilized to interpret an ambiguous streaming/bouncing display when combined with a visual signal; their results have been replicated and supported in later studies (Bushara et al., 2003; Scheier, Lewkowics, \& Shimojo, 2003; Watanabe \& Shimojo, 2001a, 2001b). Recently, Shimojo et al. (2001) suggested that multimodal attention might be involved in the perception of streaming/bouncing displays; multimodal transient signals, such as auditory tones and tactile vibrations, at coincidence reliably increased the perception of bouncing. It seems that a deprivation of the attentional resources to be allocated to vision might interfere with the recruitment of local motion signals along a straight motion path.

In line with the findings above, it has been suggested that bouncing is dominantly perceived when observers track moving objects with insufficient visual attentional resources. Using a task in which observers detected motion direction in a random-dot kinematogram, Isaak and Fawcett (1997) reported the impairment of local motion integration when visual attention was distracted from the target motion. On the basis of these findings, Watanabe and Shimojo (1998) adopted a transient visual flash, as 
well as a concurrent task, at coincidence, in a streaming/ bouncing display and showed that both voluntary and involuntary distraction of visual attention away from the moving object hampered attentional tracking of the object, resulting in the perception of bouncing. In turn, these results indicate that attentional resources might be required to maintain an intact motion trajectory.

It remains unclear, however, whether the presence of external events at coincidence can alter the appearance of a streaming/bouncing display even when visual cues to distinguish the two moving objects are introduced. In daily life, each object has unique feature information, such as luminance, color, orientation, spatial frequency, and so on. Hence, to clarify the mechanism of spatiotemporal correspondence of a moving object, it is important to examine the appearance of streaming/bouncing displays under conditions in which each object has peculiar visual features. Bertenthal et al. (1993) revealed that differences in the arrangement of random-dot textures or depth from binocular disparity between moving objects increased the frequency of the perception of streaming. However, the interaction between visual cues and events at coincidence has yet to be determined.

In this study, we attempted to determine whether orientation cues could prevent the events at coincidence from biasing the perception of bouncing in streaming/bouncing displays. In previous studies, co-alignment of the orientation of moving objects with their motion axes enhanced spatiotemporal integration of local motion across frames (Verghese, Mckee, \& Grzywacz, 2000; Werkhoven, Snipe, \& Koenderink, 1990). That is, objects with an orientation parallel to their motion path can be detected more easily than those oriented in an orthogonal direction. This enhanced detection of objects parallel to their motion path might arise from an elongated visual field, which is sensitive to parallel orientation (Fredericksen, Verstraten, \& van de Grind, 1994; Polat \& Norcia, 1998; Werkhoven et al., 1990), or from a network that enhances motion processing along the same path (Verghese et al., 2000). Therefore, we hypothesized that parallelism of moving objects with their motion path would increase the perception of streaming, because strong integration of local motion signals should occur along a straight motion path. On the other hand, the perception of bouncing should be dominantly reported when the orientation of moving objects is orthogonal to the motion path, because in this situation, weak integration of motion signals along the straight path is expected.

We also tried to clarify whether or not maintenance of enhanced integration of local motion with an object parallel to its motion path requires attentional resources. In previous studies, the perception of bouncing increased when a transient auditory signal was salient enough to attract observers' attention (Watanabe \& Shimojo, 2001b). These results suggest that temporal assignment of multimodal attention to the transient tone hampers visual processing. Spatiotemporal integration of local motion signals might be blocked when attentional resources are shifted from vision to audition (Watanabe, 2001), resulting in a hindrance of the correspondence of objects in a straight motion path and, finally, the perception of bouncing motion. Therefore, presentation of a transient tone at coincidence enables us to examine the role of multimodal attention in the integration of visual local motion. However, since the moving objects in the previous studies were in the form of a simple and uniform geometrical shape, such as a circle or a square, it was unclear whether the enhanced motion integration by parallel orientation of the moving object to its motion path could be altered by decreasing the visual attentional resources devoted to the moving object at coincidence.

Attention modulation during a single modality (i.e., vision) should also be examined. In this study, we presented with a transient flash at coincidence (Experiment 2). It has been reported that a sudden onset of visual distractors can hamper performance in the change detection paradigm (O’Regan, Rensink, \& Clark, 1999). This indicates that a transient flash can attract visual attention, which is necessary for the visual system to detect image changes across frames. A similar phenomenon, called attentional capture, has also been reported; a transiently presented object attracts visual attention (Yantis \& Jonides, 1984). We also used a dual-task paradigm to distract visual attention from moving objects at coincidence (Experiment 3 ). It goes without saying that in the paradigm, the central task can diminish performance in the peripheral task (e.g., Braun, \& Sagi, 1990; Joseph, Chun, \& Nakayama, 1997). By examining the effect of visual attention modulation on the spatiotemporal integration of moving objects, we sought to determine whether enhancement of spatiotemporal integration demands visual attentional resources and what kind of attention, multimodal or unimodal, can strongly affect perception of streaming/bouncing motion displays.

\section{EXPERIMENT 1}

\section{Method}

Apparatus. A personal computer (Sony Vaio) was used to control the presentation of stimuli and the collection of data. The stimuli were displayed on a 19-in. CRT monitor (Nanao, FlexScan T761) with a resolution of $1,024 \times 768$ pixels. Using the software for gamma correction and a luminance meter (Minolta, LS-100), we linearized the luminance value of the CRT display. The transient tone was emitted from a PC speaker that was placed in front of the participants but was invisible because of the darkness of the experimental room.

Participants. Five naive individuals participated in this experiment; all were unaware of the purpose of the experiment. They had normal or corrected-to-normal visual acuity. After reading a document detailing the experimental procedures, informed consent was obtained from each participant. The experimental procedures were consistent with the Declaration of Helsinki.

Stimuli. A schematic representation of an example motion sequence is shown in Figure 1A. The moving objects were in the form of Gaussian-modulated sinusoidal patches (Gabors) with a spatial frequency of $1.5 \mathrm{cpd}$ (cycles per degree), the standard deviation of the Gaussian modulation was $0.165^{\circ}$ of visual angle, and the luminance contrast of the Gabors was 0.3. To keep the two objects symmetrical along the vertical axis, the phase of sinusoidal patches was set to $270^{\circ}(3 / 2 \pi)$. Gabors were presented on a neutral gray background with a luminance of $38 \mathrm{~cd} / \mathrm{m}^{2}$. 
A

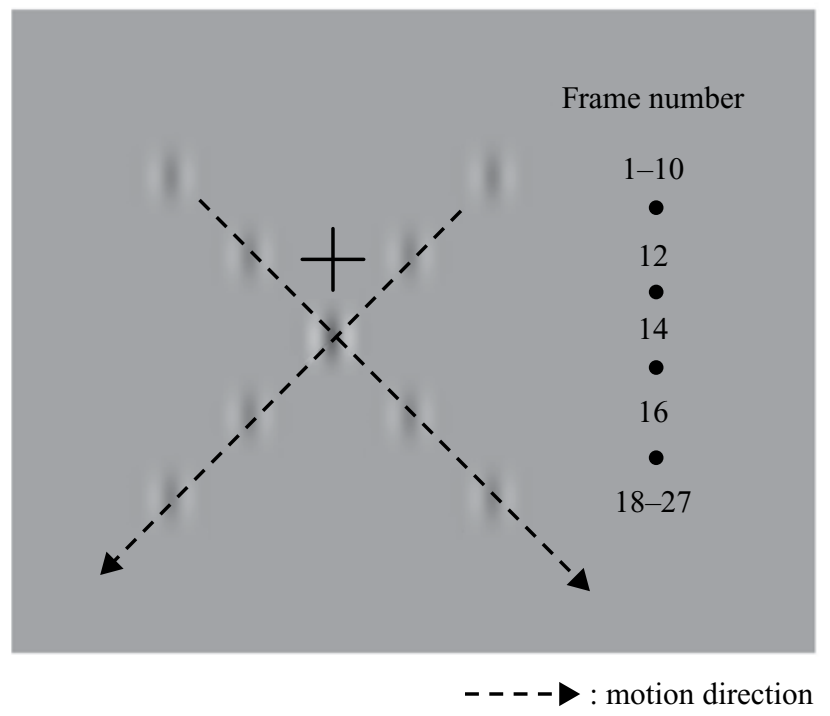

B

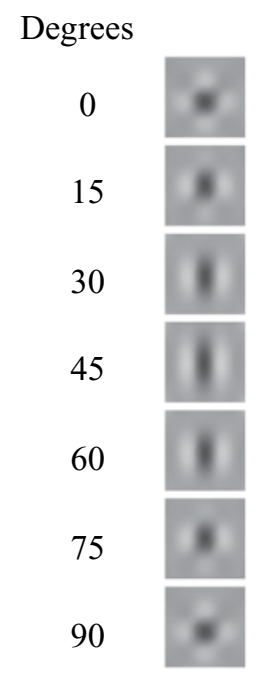

Figure 1. (A) Schematic representation of the motion sequence used in Experiment 1. Arrows indicate the motion direction of each Gabor. (B) The appearance of superposition of two moving Gabors (Frame 14) for each orientation condition.

The two Gabors statically appeared for 10 frames $(26 \mathrm{msec}$ per frame $\times 10$ ) on opposite sides of the display, separated by $2.66^{\circ}$ and $0.99^{\circ}$ above the fixation cross $\left(0.66^{\circ}\right.$ in length and $0.04^{\circ}$ in width, with a luminance of $0.3 \mathrm{~cd} / \mathrm{m}^{2}$ ). From Frames 11 to 18 , the two Gabors diagonally moved toward the center of the display at a steady speed $\left(0.47^{\circ}\right.$ per frame, $\left.18 \mathrm{deg} / \mathrm{sec}\right)$, with superposition occurring $0.99^{\circ}$ below the fixation cross in Frame 14 (hereafter, the momentary superposition will be referred to as coincidence). At coincidence, the luminance modulation of the Gabors, not the luminance of them itself, was added to each other; hence, luminance contrast, not variation in mean luminance, was changed (the maximal value of luminance contrast was 0.6 at complete superposition). Several snapshots of the superposition of Gabors at coincidence (Frame 14) are shown in Figure 1B. From Frames 19 to 27, the two Gabors were paused, and on Frame 28, they disappeared.

Orientation differences between the Gabors and their motion axes were then introduced (Figure 2A). Complete alignment was designated as $0^{\circ}$, and complete misalignment as $90^{\circ}$. Within the range of $0^{\circ}-90^{\circ}$, seven orientation differences were set $\left(0^{\circ}, 15^{\circ}, 30^{\circ}, 45^{\circ}, 60^{\circ}\right.$, $75^{\circ}$, and $\left.90^{\circ}\right)$. In this experiment, a transient tone at coincidence was also introduced. The duration, sound pressure level, and pitch of the tone were $8 \mathrm{msec}, 70 \mathrm{~dB}$ at the ear of the participants, and $1800 \mathrm{~Hz}$, respectively. There was background noise of about $35 \mathrm{~dB}$.

Procedures. The participants sat at a distance of $93 \mathrm{~cm}$ from the CRT display in a darkened room. Their heads were positioned on a chin- and headrest to fix the visual field. During each trial, they were asked to report which motion trajectory, streaming or bouncing, they perceived. Each response was recorded by pressing assigned keys on the computer keyboard. They were asked to make each response carefully but not urgently. After each response, the next trial was immediately started. The participants received a training session to familiarize them with the task in each condition in the presence/ absence of a tone. In the experimental session, trials were divided into two blocks according to the presence/absence of the transient tone; in each block, the order of the trials was randomized. Each block consisted of 70 trials ( 7 trials involving orientation differences between the Gabors and their motion axes $\times 10$ replications). As a result, the participants underwent 140 trials (70 trials $\times 2$ blocks $)$ in total.

\section{Results}

The averaged results for all the participants are plotted in Figure 2B as a function of the orientation differences between the Gabors and their motion axes and the presence/absence of the transient tone at coincidence. The results were statistically assessed using a two-way repeated measures ANOVA with orientation differences and the presence/absence of the tone as factors. The main effect of the orientation differences was significant $[F(6,24)=$ 28.635, $p<.0001$ ]. Multiple comparison tests (Ryan's method) showed the following: At an orientation of $0^{\circ}$, the results were significantly different from those at $30^{\circ}$, $45^{\circ}, 60^{\circ}, 75^{\circ}$, and $90^{\circ}(t=5.051,6.037,8.624,8.501$, and 7.885 , respectively; $p<.05$ ); those at $15^{\circ}$ were significantly different from those at $30^{\circ}, 45^{\circ}, 60^{\circ}, 75^{\circ}$, and $90^{\circ}$ $(t=5.175,6.16,8.747,8.624$, and 8.008, respectively; $p<.05)$; and those at $30^{\circ}$ were significantly different from those at $60^{\circ}$ and $75^{\circ}(t=3.573$ and 3.450, respectively; $p<.05)$. The main effect of the presence/absence of the transient tone was also significant $[F(1,5)=9.353, p<$ $.04]$. On the other hand, the interaction between the two factors was not significant $[F(6,30)=1.206, p>.3]$.

\section{Discussion}

In this experiment, we investigated how a transient tone that would distract multimodal attention away from vision affected the spatiotemporal integration of local motion signals along a straight path. Consequently, we observed that the two experimental variables, the presence/absence of a transient tone at coincidence and the collinearity of Gabors with their motion axes, simultaneously contributed to the perception of streaming/bouncing.

As has been described in the introduction, it is necessary to examine whether the effect of a transient flash 

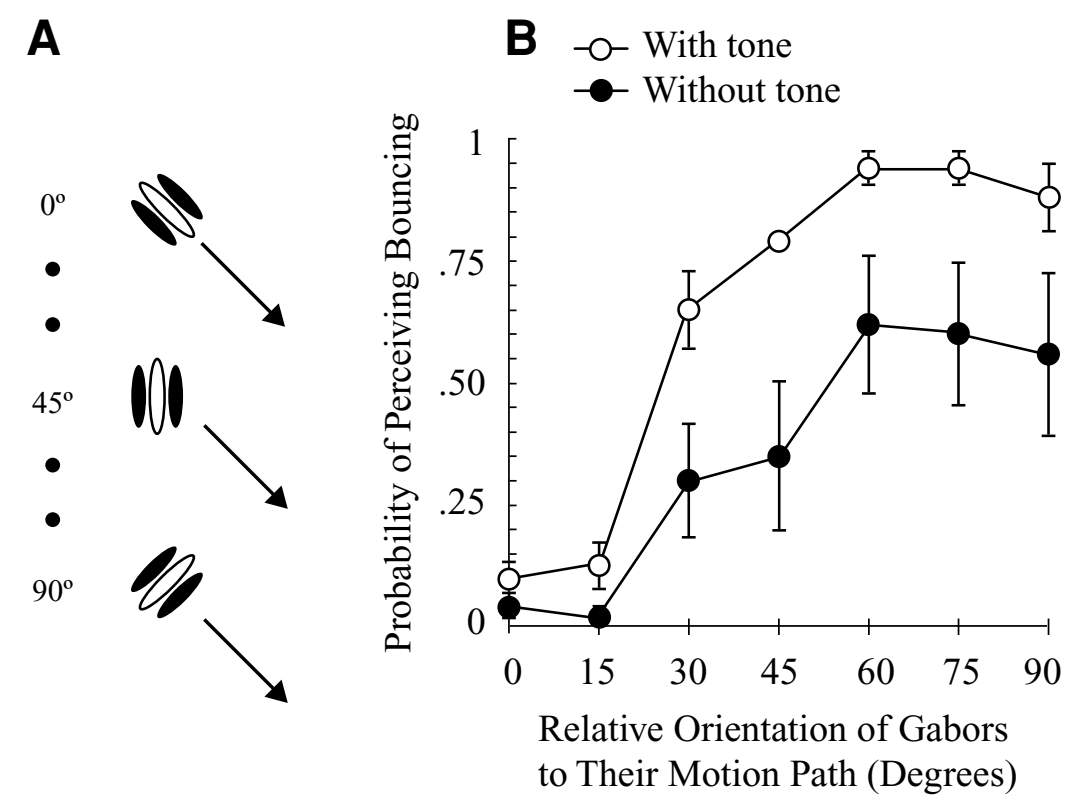

Figure 2. (A) Schematic representation of the orientation difference between a Gabor and the motion direction. (B) The results of Experiment $1(N=5)$. White and black symbols represent the probability of a bouncing response in the presence/absence of a tone, respectively. Error bars represent the standard errors of the means.

on streaming/bouncing perception is similar to that of the transient tone employed in Experiment 1. Watanabe (2001) suggested that a transient tone can distract multimodal attention away from vision. In this situation, to judge the appearance of a streaming/bouncing display, observers must immediately return their attention to vision after abrupt onset of the tone. Recently, it has been suggested that dividing an observer's attention between two different modalities (e.g., vision and audition) is more difficult than dividing attention within a single modality (Spence \& Reed, 2003). Therefore, it was unclear whether distraction of unimodal attention by the presentation of a transient flash would be as strong as that by presentation of a transient tone in Experiment 1. In previous studies, Watanabe and Shimojo (1998) showed that the sudden onset of distractors can distract visual attention away from a moving object, resulting in a significant increase in the perception of bouncing (see also Burns \& Zanker, 2000).

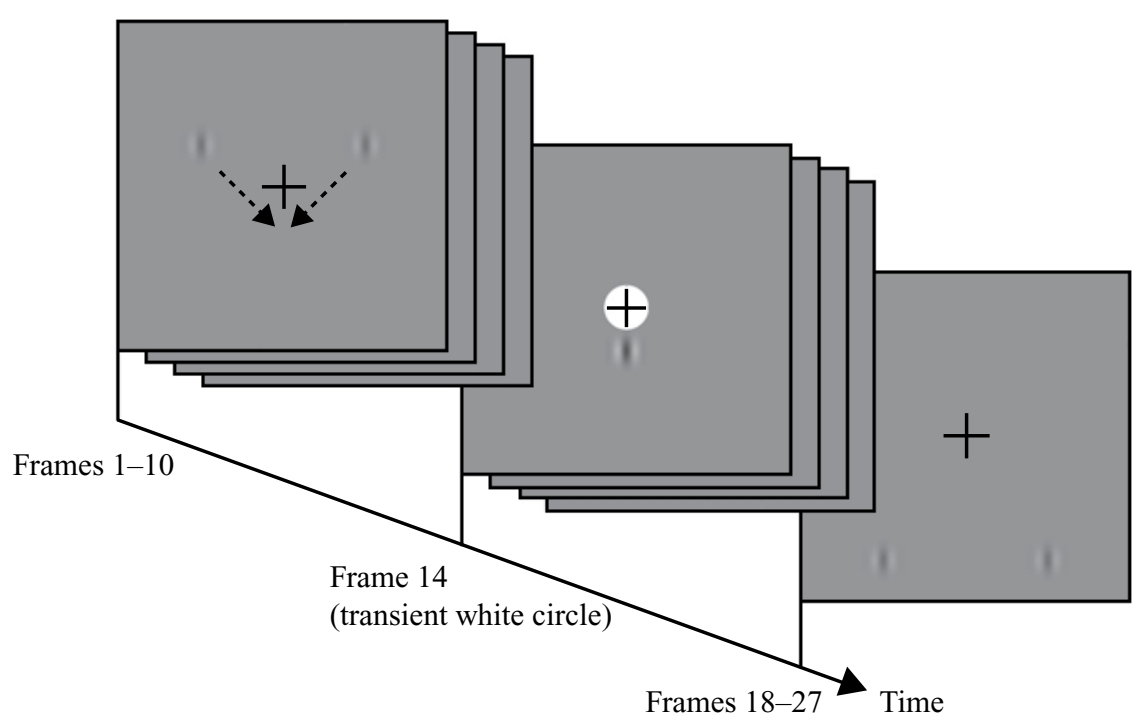

Figure 3. Schematic representation of the motion sequence used in Experiment 2. In Frame 14, a white circle was transiently presented behind the fixation cross. 
In the following experiments, we investigated the potential effects of distracting visual attention away from the object during perception of streaming/bouncing where the Gabor orientation was modulated as in Experiment 1.

\section{EXPERIMENT 2}

\section{Method}

Apparatus. The same apparatus as that in Experiment 1 was used in Experiment 2.

Participants. Five individuals participated in Experiment 2. Of these, 3 also had participated in Experiment 1. All had no knowledge of the experiment.

Stimuli. The stimuli used were basically the same as those used in Experiment 1, except for the following: A transient flash in the form of a visual circle was included at coincidence (Frame 14), instead of a transient tone (Figure 3). The size and luminance of the circle were $0.66^{\circ}$ and $76 \mathrm{~cd} / \mathrm{m}^{2}$, respectively.

Procedure. The procedure was the same as that in Experiment 1, except for the following. The participants received a training session to familiarize them with the tasks of each condition in the presence/ absence of the transient circle. During the experimental session, the presence/absence of a transient flash divided the trials into two blocks. In each block, the order of the trials was randomized. Each block consisted of 70 trials, and as a result, each observer underwent 140 trials in total

\section{Results}

The averaged results for all the participants are plotted in Figure 4 as a function of the orientation differences between the Gabors and their motion axes and the presence/ absence of the transient flash at coincidence. The results were statistically assessed using a two-way repeated measures ANOVA with seven orientation differences and the presence/absence of the flash as factors.

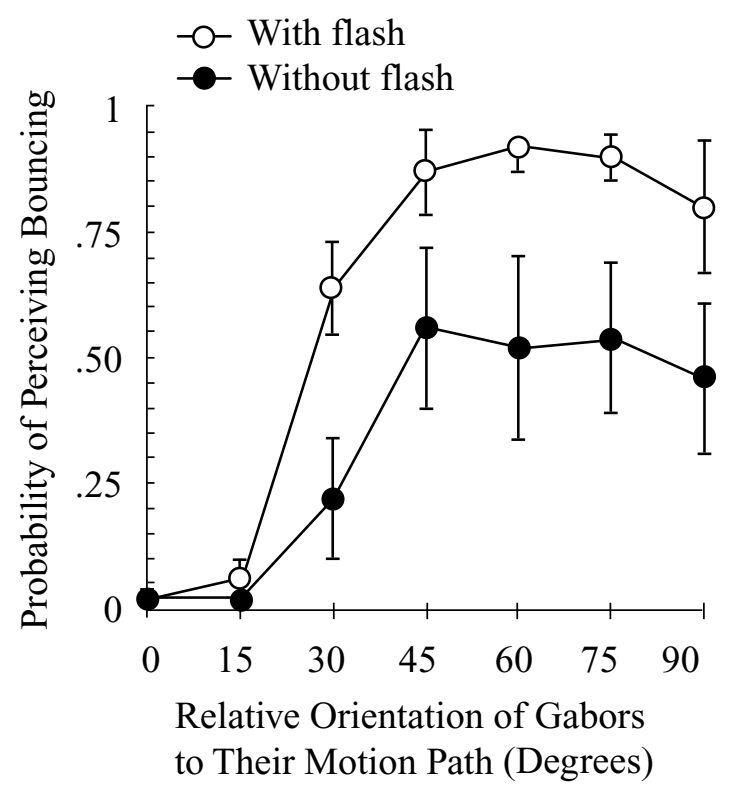

Figure 4. The results of Experiment $2(N=5)$. White and black symbols represent the probability of a bouncing response in the presence/absence of a flash, respectively. Error bars represent the standard errors of the means.
The main effect of the orientation differences was significant $[F(6,24)=35.865, p<.0001]$. Multiple comparison tests showed the following: At an orientation of $0^{\circ}$, the results were significantly different from those at $30^{\circ}, 45^{\circ}, 60^{\circ}, 75^{\circ}$, and $90^{\circ}(t=5.492,9.310,9.377,9.377$, and 8.171 , respectively; $p<.05)$; those at $15^{\circ}$ were significantly different from those at $30^{\circ}, 45^{\circ}, 60^{\circ}, 75^{\circ}$, and $90^{\circ}(t=5.224,9.942,9.109,9.109$, and 7.904, respectively; $p<.05)$; and those at $30^{\circ}$ were significantly different from those at $45^{\circ}, 60^{\circ}, 75^{\circ}$, and $90^{\circ}(t=3.818$, $3.885,3.885$, and 2.679 , respectively; $p<.05)$. The main effect of the presence/absence of the transient flash was marginally significant $[F(1,4)=6.742, p=.0603]$, and the interaction between the two factors was significant $[F(6,24)=2.998, p<.03]$. The simple effect showed that the presence/absence of the transient flash was significant with all the orientation differences $(p<.03)$, except for at $0^{\circ}$ and $15^{\circ}[F(1,28)=0$ and .77 , respectively; $p>.05]$.

\section{Discussion}

Experiment 2 showed the following two findings. First, the shift of visual attention with the transient flash could generally increase the bias toward bouncing, which is consistent with the results of previous studies (Burns \& Zanker, 2000; Watanabe \& Shimojo, 1998). Second, when the transient flash was presented, the perception of bouncing did not increase when the Gabors were aligned with their motion path $\left(0^{\circ}\right.$ and $\left.15^{\circ}\right)$. The latter results were interpreted as the effect of spatiotemporal integration of motion signals along a straight path, which maintained an intact motion trajectory.

In the next experiment, we attempted to confirm whether the effect of spatiotemporal integration of motion signals could work even when visual attention was voluntarily distracted away from the moving objects.

\section{EXPERIMENT 3}

\section{Method}

Apparatus. The same apparatus as that in Experiments 1 and 2 was used in Experiment 3.

Participants. Five individuals participated in this experiment; all of them had also participated in Experiment 2 and had no knowledge of the experiment.

Stimuli. The stimuli were basically the same as those used above, except for the following: In this experiment, we adopted a transient gap in a bull's-eye fixation symbol at coincidence (Figure 5). The size of the gap was $0.66^{\circ} \times 0.11^{\circ}$. The luminance of the gap was $38 \mathrm{~cd} / \mathrm{m}^{2}$, which was equivalent to the background. The gap was presented on either the left or the right of the bull's-eye.

Procedure. The procedure was the same as that in Experiment 2, except for the following. The participants received a training session to familiarize them with the tasks, with and without the identification of gap position. During the experimental session, the presence/ absence of the concurrent task divided the trials into two blocks. In the concurrent task block - that is, the dual-task block - the participants judged which side of the bull's-eye had a gap and whether the motion trajectory was streaming or bouncing. They were encouraged to accurately respond to the position of the transient gap and were told that they would have to repeat the same block if the percentage of correct results was not above $90 \%$. In the single-task block, the participants were asked to ignore the presence of the gap 


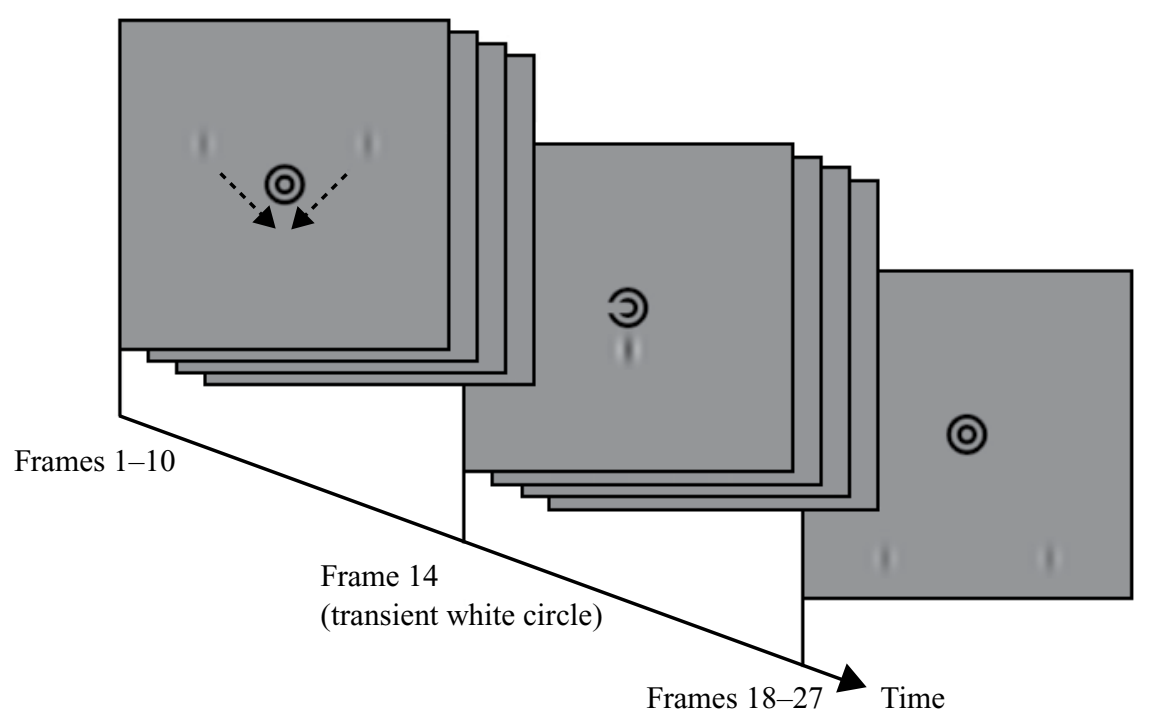

Figure 5. Schematic representation of the motion sequence used in Experiment 3. In Frame 14, a bull's-eye was transiently presented behind the fixation cross.

in the bull's-eye; therefore, they reported on the perceived motion trajectory only. Each block consisted of 70 trials, and as a result, the participants received 140 trials in total, as above. In each block, the order of the trials was randomized.

\section{Results}

The averaged results for all the participants are plotted in Figure 6 as a function of the orientation differences between the Gabors and their motion axes and the dual/ single task at coincidence. The results were statistically assessed using a two-way repeated measures ANOVA with orientation differences and the dual/single task as factors. The main effect of the orientation differences was significant $[F(6,24)=10.346, p<.0001]$. Multiple comparison tests showed the following: at an orientation of $0^{\circ}$, the results were significantly different from those at $30^{\circ}$, $45^{\circ}, 60^{\circ}, 75^{\circ}$, and $90^{\circ}(t=2.767,4.759,4.759,4.759$, and 4.267 , respectively; $p<.05)$; and those at $15^{\circ}$ were significantly different from those at $30^{\circ}, 45^{\circ}, 60^{\circ}, 75^{\circ}$, and $90^{\circ}$ $(t=3.099,5.091,5.091,5.091$, and 4.599, respectively; $p<.05)$. The main effect of the dual/single task was also significant $[F(1,4)=16.879, p<.02]$, as was the interaction between the two factors $[F(6,24)=4.025, p<.007]$. The simple effect showed that the effect of the dual/single tasks was significant with all the orientation differences $(p<.05)$, except for $0^{\circ}$ and $15^{\circ}[F(1,28)=0.761$ and 0.487 , respectively; $p>.05]$.

\section{Discussion}

The results show that the enhanced spatiotemporal integration of local motion signals of objects parallel to their motion path persisted even when visual attention was voluntarily distracted away from the moving object.

We do not think that the transient appearance of the gray bar on the bull's-eye increased the perception of bouncing. In this experiment, the observers were instructed first to identify the position of the bar or ignore the bar and, second, to report whether streaming or bouncing was perceived. Therefore, it is possible that not only the voluntary attention shift to the fixation point, but also the involuntary attention shift due to abrupt onset of the gray bar contributed to the increased perception of bouncing. However, since the probability of perceiving bouncing in the ignore condition was not as great as in the nonflash condition in Experiment 2, we believe that the effect of the abrupt onset of the gray bar on the perception of the streaming/bouncing display was very small.

The bouncing responses were less frequent in the singletask condition in this experiment than in those in Experiments 1 and 2. A statistical analysis showed that the overall proportions of bouncing responses in this experiment were significantly lower than those in Experiment $2[t(4)=$ $2.79, p<.05]$. We speculate that the outcome might stem from the difference in the attentional set of the observers between the two experiments. In this experiment, the observers could expect the appearance of a gap in the fixation symbol and, thus, direct their attention toward moving objects, as well as the symbol, in advance. In contrast, the observers could not attend to the flash stimulus in Experiment 2 , because the flash was suddenly and transiently presented at coincidence. Hence, attentional tracking of moving objects, which is required for streaming perception, was likely to be hampered more in Experiment 2 than in this experiment. Consequently, the bouncing responses in this experiment appeared to be relatively reduced, in comparison with those in Experiment 2.

\section{GENERAL DISCUSSION}

In this study, we investigated the role of spatiotemporal integration of local motion signals and multimodal or visual attention on the perception of streaming/bouncing displays. In previous studies, a transient auditory signal at co- 


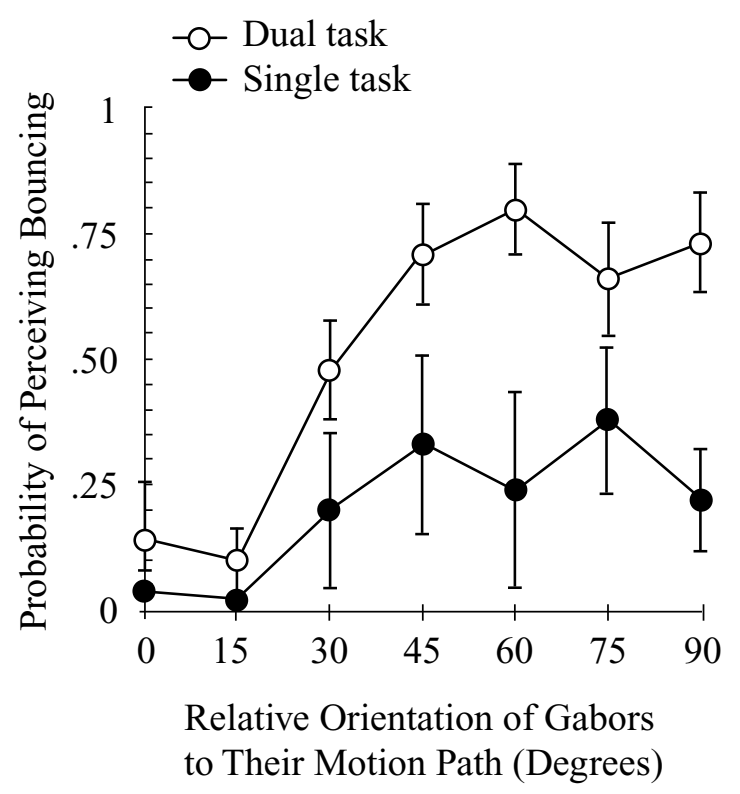

Figure 6. The results of Experiment $3(N=5)$. White and black symbols represent the probability of a bouncing response under dual-/single-task conditions, respectively. Error bars represent the standard errors of the means.

incidence biased the perception toward bouncing (Sekuler et al., 1997; Watanabe \& Shimojo, 2001a, 2001b), as did distraction of visual attention (Burns \& Zanker, 2000; Watanabe \& Shimojo, 1998). However, in these previous studies, since a uniform geometric object was used as the moving object, it was unclear how the motion integration mechanism interacted with the modulation of attention. In the present study, by modulating the orientation differences between moving Gabors and their motion axes, we investigated the role of relative orientation to the motion axis in the perception of streaming/bouncing motion displays. In the previous studies, it has been suggested that spatiotemporal integration is enhanced when the moving object is parallel, rather than orthogonal, to its motion path (Verghese et al., 2000; Werkhoven et al., 1990). We expected the dominant perception of streaming with parallel or near parallel (less than $30^{\circ}$ ) conditions, because the spatiotemporal integration of motion signals along a straight path should be enhanced. On the other hand, we predicted that perception would dominantly result in the perception of bouncing in other conditions, because the integration should be weak. In our hypothesis, we also associated the effect of modulation of attention on spatiotemporal motion integration. In the parallel condition, the effect of attention should not be so strong as to change the perception from streaming to bouncing, because the integration was expected to be strong enough to retain the intact trajectory. On the other hand, in the orthogonal condition, the perception of bouncing should be dominant when the modulation of attention is added, because in this situation, spatiotemporal integration of motion information should be rather weak and easily modified by attention distrac- tion. By using a transient tone (Experiment 1), a transient flash (Experiment 2), and dual tasks (Experiment 3), we demonstrated that spatiotemporal integration of local motion signals along a straight trajectory strongly contributes to the perception of streaming and that enhanced integration of an object moving parallel to its path cannot be altered by various types of attention modulation. That is, the enhancement of spatiotemporal integration with Gabor orientation parallel to the motion path is tolerant of attention modulation. This also indicates the existence of motion representation, which is relatively free from the effect of attention distraction.

The results of this study clearly differentiate the contribution of spatiotemporal integration of motion signals along a straight trajectory from the feature-binding orientation and motion direction. With our stimuli, the orientation differences between Gabors were $90^{\circ}$ both when the Gabor orientation was parallel and when it was orthogonal to the motion path. The same result would be expected between the two orientation conditions if the visual system were to bind the orientation and motion direction, as has been reported in previous studies (McLeod, Driver, \& Crisp, 1988; Nakayama \& Silverman, 1986). As it was, perception of streaming was dominant in the parallel condition, whereas bouncing was dominant in the orthogonal condition. These results indicate that at least under the stimulus conditions in this study, the visual system might not utilize representation of feature binding (Paul \& Schyns, 2003; Tresiman \& Gelade, 1980) as a cue to identify moving objects. This idea might be related to previous studies, which have suggested that the binding of visual features (color) and motion direction is unexpectedly weak (Saiki, 2003a, 2003b). Therefore, we suggest that the visual system relies on lower level motion integration in determining the appearance of a streaming/bouncing motion display.

One might argue that instead of motion integration along a straight path, spatiotemporal grouping biased the responses toward streaming. Field, Hayes, and Hess (1993) showed that a contour-like structure (path) consisting of several Gabors was detected better when the Gabors were parallel, rather than orthogonal, to the motion path. They attributed these phenomena to an association field where the outputs of a neighboring filter responding to a similar orientation are integrated. Moreover, their results showed that detection of the path was hampered when the relative Gabor orientation exceeded $30^{\circ}$. The orientation limitation for detecting the path in Field et al. was also seen in the results of our study. Moreover, in Experiments 2 and 3 in the present study, distraction of attention did not have the effect of biasing responses toward bouncing when the orientation differences between the Gabors and their motion paths were $0^{\circ}$ and $15^{\circ}$. However, when the differences exceeded $30^{\circ}$, a significant effect of distraction was observed. Therefore, one might argue that the association field working in space and time (Alais \& Lorenceau, 2002; Georges, Seriès, Frégnac, \& Lorenceau, 2002) involves the effect of alignment of the moving object and its motion path. However, the same orientation filter should 
involve both motion integration and the perceptual grouping of orientation information in V1 (Field et al., 1993; Geisler, Albrecht, Crene, \& Stern, 2001). Therefore, we could not determine the effect of spatiotemporal integration of motion signals from that of grouping based on the association field; further investigations will clarify this matter in the future.

The transient tone employed in Experiment 1 might have had an effect other than modulation of multimodal attention, because of the difference in interaction seen with ANOVA analyses between Experiments 1 and 2-3. Recent studies have suggested that a transient tone at coincidence not only distracts observers' attention away from the object, but also biases judgment toward bouncing (Sanabria, Correa, Lupiáñez, \& Spence, 2004). That is, by presenting the transient tone at coincidence, the bouncing motion might be selected, due to the deprivation of the attentional resources to be allocated to vision, as well as to some kind of cognitive bias for a collision event (Guski $\&$ Troje, 2004).

It is possible that our hypothesis and explanation might conflict with conventional ideas whereby orientation orthogonal to motion direction involves motion perception through a circular aperture (Adelson \& Movshon, 1982). However, recent studies have suggested that the motion sensor, which is selective to orientation parallel with the motion axis, also plays an important role in perceiving motion direction (Geisler, 1999; Geisler et al., 2001; Jancke, 2000). Rather, parallel orientation appears to strongly contribute to the spatiotemporal integration of local motion signals along a straight motion path (Verghese et al., 2000; Werkhoven et al., 1990). In our experiments, in agreement with recent studies, parallel, rather than orthogonal, orientation to the motion axis seems critical for the stable perception of streaming. The perception of appearance in a streaming/bouncing display is based on the perception of motion direction; that is, observers must report whether the motion direction before coincidence changes after coincidence. Since it is assumed that an elongated receptive field is precisely in tune with motion direction (Werkhoven et al., 1990), objects moving parallel, rather than orthogonal, to the motion path might dominantly act to reduce the perception of bouncing, because the detection of motion is likely to be more precise in the parallel than in the orthogonal orientation condition.

We conclude that strong spatiotemporal integration of local motion signals along a straight path can contribute to the perception of streaming. Moreover, this enhanced spatiotemporal integration could not be altered by the modulation of attention, indicating the possibility that motion integration of a target parallel to its motion path across the frame is relatively free from the effect of attention distraction.

\section{REFERENCES}

Adelson, E. H., \& Bergen, J. R. (1985). Spatio-temporal energy models for the perception of motion. Journal of the Optical Society of America A, 2, 284-299.
Adelson, E. H., \& Movshon, J. A. (1982). Phenomenal coherence of moving visual patterns. Nature, 300, 523-525.

Alais, D., \& LoREnceaU, J. (2002). Perceptual grouping in the Ternus display: Evidence for an "association field" in apparent motion. Vision Research, 42, 1005-1016.

Anstis, S. M., \& RAMAchandRan, V. S. (1986). Entrained path deflection in apparent motion. Vision Research, 26, 1731-1739.

Bertenthal, B. I., Banton, T., \& Bradbury, A. (1993). Directional bias in the perception of translating patterns. Perception, 22, 193-207.

Braun, J., \& SaGi, D. (1990). Vision outside the focus of attention. Perception \& Psychophysics, 48, 45-58.

BuRNS, N. R., \& ZANKER, J. M. (2000). Streaming and bouncing: Observations on motion defined objects. Clinical \& Experimental Ophthalmology, 28, 220-222.

Bushara, K. O., HanaKawa, T., Immisch, I., Toma, K., KansaKu, K., \& Hallett, M. (2003). Neural correlates of cross-modal binding. Nature Neuroscience, 6, 190-195.

Cavanagh, P. (1992). Attention based motion perception. Science, 257, 1563-1565.

Field, D. J., Hayes, A., \& Hess, R. F. (1993). Contour integration by the human visual system: Evidence for a local "association field." Vision Research, 33, 173-193.

Fredericksen, R. E., Verstraten, F. A., \& van de Grind, W. A. (1994). Spatial summation and its interaction with the temporal integration mechanism in human motion perception. Vision Research, 34, 3171-3188.

GEISLER, W. S. (1999). Motion streaks provide a spatial code for motion direction. Nature, 400, 65-69.

Geisler, W. S., Albrecht, D. G., Crane, A. M., \& Stern, L. (2001). Motion direction signals in the primary visual cortex of cat and monkey. Visual Neuroscience, 18, 501-516.

Georges, S., Seriès, P., Frégnac, Y., \& Lorenceau, J. (2002). Orientation dependent modulation of apparent speed: Psychophysical evidence. Vision Research, 42, 2757-2772.

Gepshtein, S., \& Kubovy, M. (2000). The emergence of visual objects in space-time. Proceedings of the National Academy of Sciences, 97, 8186-8191.

GoldberG, D. M., \& Pomerantz, J. R. (1982). Models of illusory pausing and striking. Journal of Experimental Psychology: Human Perception \& Performance, 8, 547-561.

Guski, R., \& TROJE, N. F. (2003). Audiovisual phenomenal causality. Perception \& Psychophysics, 65, 789-800.

IsAaK, M. I., \& FAWCETT, S. L. (1997). Attention facilitates sequential recruitment in the perception of motion direction. Perception, 26 (Suppl.), 96.

JANCKE, D. (2000). Orientation formed by a spot's trajectory: A twodimensional population approach in primary visual cortex. Journal of Neuroscience, 20, 1-6.

Joseph, J. S., Chun, M. M., \& Nakayama, K. (1997). Attentional requirements in a "preattentive" feature search task. Nature, 387, 805807.

Lu, Z.-L., \& SperLing, G. (2001). The three systems theory of human visual motion perception: Review and update. Journal of the Optical Society of America A, 18, 2331-2370.

MCLeod, P., Driver, J., \& CRISP, J. (1988). Visual search for a conjunction of movement and form is parallel. Nature, 332, 154-155.

NAKAYAma, K., \& Silverman, G. H. (1986). Serial and parallel processing of visual feature conjunctions. Nature, 320, 264-265.

O’Regan, J. K., Rensink, R. A., \& Clark, J. J. (1999). Change-blindness as a result of "mudsplashes." Nature, 398, 34.

Paul, L., \& Schyns, P. G. (2003). Attention enhances feature integration. Vision Research, 43, 1793-1798.

Polat, U., \& NorCia, A. (1998). Elongated physiological summation pools in the human visual cortex. Vision Research, 38, 3735-3741.

PyLYShyn, Z. W., \& StoRM, R. W. (1988). Tracking multiple independent targets: Evidence for a parallel tracking mechanism. Spatial Vision, 3, 179-197.

SAIKI, J. (2003a). Feature binding in object-file representations of multiple moving items. Journal of Vision, 3, 6-21.

SAIKI, J. (2003b). Spatiotemporal characteristics of dynamic feature binding in visual working memory. Vision Research, 43, 2107-2123. 
Sanabria, D., Correa, A., Lupiáñez, J., \& Spence, C. (2004). Bouncing or streaming? Exploring the influence of auditory cues on the interpretation of ambiguous visual motion. Experimental Brain Research, 157, 537-541.

Scheier, C., Lewkowics, D. J., \& Shimojo, S. (2003). Sound induces perceptual reorganization of an ambiguous motion display in human infants. Developmental Science, 6, 233-244.

Sekuler, R., Sekuler, A. B., \& Lau, R. (1997). Sound alters visual motion perception. Nature, $\mathbf{3 8 5}, 308$.

Shimojo, S., Scheier, C., Nijhawan, R., Shams, L., Kamitani, Y., \& Watanabe, K. (2001). Beyond perceptual modality: Auditory effects on visual perception. Acoustical Science \& Technology, 22, 61-67.

Shimojo, S., \& Sham, L. (2001). Sensory modalities are not separate modalities: Plasticity and interactions. Current Opinion in Neurobiology, 11, 505-509.

Spence, C., \& ReAd, L. (2003). Speech shadowing while driving: On the difficulty of splitting attention between eye and ear. Psychological Science, 14, 251-256.

Sumi, S. (1995). Bounce effect seen in opposite motions. Japanese Psychological Research, 37, 195-200.

Tresiman, A., \& Gelade, G. (1980). A feature integration theory of attention. Cognitive Psychology, 12, 97-136.

Verghese, P., McKee, S. P., \& Grzywarc, N. M. (2000). Stimulus configuration determines the detectability of motion signals in noise. Journal of the Optical Society of America A, 17, 1525-1534.
Verstraten, F. A. J., Cavanagh, P., \& Labianca, A. T. (2000). Limits of attentive tracking reveal temporal properties of attention. Vision Research, 40, 3651-3664.

Watamaniuk, S. N. J., McKee, S. P., \& Grzywacz, N. M. (1995). Detecting a trajectory embedded in random-direction motion noise. Vision Research, 35, 65-77.

WatanaBe, K. (2001). Crossmodal interaction in humans. Unpublished doctoral dissertation, California Institute of Technology.

Watanabe, K., \& Shimojo, S. (1998). Attentional modulation in perception of visual motion events. Perception, 27, 1041-1054.

Watanabe, K., \& Shimojo, S. (2001a). Postcoincidence trajectory duration affects motion event perception. Perception \& Psychophysics, 63, 16-28.

Watanabe, K., \& Shimojo, S. (2001b). When sound affects vision: Effects of auditory grouping on visual motion perception. Psychological Science, 12, 109-111.

Werkhoven, P., Snipe, H. P., \& Koenderink, J. J. (1990). Effects of element orientation on apparent motion perception. Perception \& Psychophysics, 47, 509-525.

YAnTIS, S., \& Jonides, J. (1984). Abrupt visual onsets and selective attention: Evidence from visual search. Journal of Experimental Psychology: Human Perception \& Performance, 10, 601-621.

(Manuscript received August 20, 2004;

revision accepted for publication August 2, 2005.) 